\title{
Experiments on the CMB Spectrum, Big Jets Model and Their Implications for the Missing Half of the Universe
}

\author{
Leonardo $\mathrm{Hsu}^{1, \star}$ and Jong-Ping $\mathrm{Hsu}^{2, \star \star}$ \\ ${ }^{1}$ College of Education and Human Development, University of Minnesota, Minneapolis, MN 55455, USA \\ ${ }^{2}$ Department of Physics, University of Massachusetts Dartmouth, North Dartmouth, MA 02747, USA
}

\begin{abstract}
Based on the limiting continuation of Lorentz-Poincaré invariance, we propose an alternative formulation of the generalized Planck distribution for inertial and noninertial frames. The Lorentz invariant Planck distribution law leads to a new physical interpretation of the dipole anisotropy of the Cosmic Microwave Background. The Big Jets model predicts a distant 'antimatter blackbody,' whose radiations could make 50\% of the sky very slightly warmer than the isotropic CMB temperature $T_{C M B}$ with a cosine function. The other $50 \%$ of the sky has the same isotropic temperature $T_{C M B}$. Thus, we could have a pseudo-dipole anisotropy because the microwaves emitted from the antimatter blackbody are totally absorbed by our matter blackbody. We suggest that accurate data of satellite experiments might be used to search for the pseudo-dipole anisotropy and the missing half of the antimatter universe.
\end{abstract}

\section{Introduction}

We discuss the concept of Lorentz-Poincaré invariance and its limiting continuation, which could be considered as a minimum generalization of the principle of relativity from inertial to non-inertial frames[1, 2]. Such a limiting Lorentz-Poincaré invariance enables us to obtain physical laws for a 'general frame' (inertial and non-inertial). As a result, we propose an alternative formulation of the generalized invariant Planck distribution that leads to a new physical interpretation of the dipole anisotropy of the Cosmic Microwave Background (CMB)[3, 4]. We stress that satellite measurements of the CMB might provide the data necessary for testing different formulations of the Planck distribution law, which is associated with different transformations of the temperature. Such a test is significant because the alternative invariant Planck distribution implies that, if there is a temperature difference (or asymmetry) between two opposite directions in sky with the pattern, $T(\theta)=T_{C M B}+T_{e} \cos \theta$, for $\cos \theta>0$, and $T(\theta)=T_{C M B}$, for $\cos \theta<0 .{ }^{1}$ Big Jets model suggests that a pseudo-dipole anisotropy could be caused by the presence of an extremely distant antimatter-blackbody.[5]

We note that satellite data from the COBE, WMAP, and Planck satellite experiments all show a six-month variation corresponding to the Earth's orbital motion. However, this effect is normally considered an undesirable source of noise in the signal so that it is typically removed from the data without

\footnotetext{
^e-mail: 1hsu@umn.edu

$\star \star$ e-mail: jhsu@umassd.edu

${ }^{1}$ The pattern $T_{e} \cos \theta$ and zero for two opposite hemispheres shows a pseudo-dipole anisotropy and is the characteristics of the total absorption of microwaves emitted from the antimatter blackbody by our matter-blackbody.
} 
much comment. It appears that there is no published data on the magnitude of this variation.[3] The magnitude of this variation is certainly detectable by satellite experiments. It can also be used to test the law of blackbody radiation in a rotational frame and the alternative 'general-frame' (GF) covariant blackbody distribution.[6] However, one must be more careful. As indicated by the analysis of the Wilson experiment[7], it is not correct to use the Lorentz transformations to analyze situations involving non-inertial circular motion, even locally (irrespective of the fact that it sometimes gives the correct answer, as in the case of lifetime dilations of unstable particles in a circular storage ring). Instead, we must first correctly generalize the blackbody distribution law to a rotational frame.

\section{Lorentz invariant blackbody distribution law}

To accomplish this, we first generalized the original Planck distribution law $P(\omega, T)=$ $1 /\left[\exp \left(\omega / k_{B} T\right)-1\right]$ for the rest frame of the blackbody to the Lorentz invariant blackbody distribution law for all inertial frames. We propose that the quantity $\omega=k_{0}$ should be replaced by the product of the four-velocity of the thermo-system $U^{\mu}=d x^{\mu} / d s$ with the wave four-vector $k^{\mu}=\left(k_{0}, k_{x}, k_{y}, k_{z}\right)$. In the rest frame of a thermo-system, its four-velocity is $U^{\mu}=(1,0,0,0)$, so $k_{\mu} U^{\mu}=k_{0}=\omega$, where $c=\hbar=1$. Thus, we insure that this new formulation remains consistent with all experimental results, as well as remains consistent with the framework of special relativity. Because Planck's law was devised before the advent of special relativity, one does not necessarily expect its original formulation to be relativistically consistent, and such a replacement is natural. We have also assumed that the quantity $k_{B} T$ is Lorentz-invariant. Although the universality of the Boltzmann constant $k_{B}$ has never been established in a relativistic framework, such an assumption is consistent with many previous discussions of the transformation properties of heat and temperature in the literature[8-13].

Thus, in this alternative formulation, the generalized Planck distribution in an inertial frame $F$ is

$$
\begin{gathered}
B\left(k_{\mu} U^{\mu}, T\right)=\frac{1}{\left[\exp \left(k_{\mu} U^{\mu}\right) /\left(k_{B} T\right)-1\right]}, \quad k_{\mu} U^{\mu}=\eta_{\mu v} k^{\mu} U^{v}, \quad T=T_{i n v}, \\
U^{\mu}=\frac{d x^{\mu}}{d s}, \quad \eta_{\mu v} U^{\mu} U^{v}=1, \quad \eta_{\mu v}=(1,-1,-1,-1), \quad c=\hbar=1,
\end{gathered}
$$

where $T$ in $B\left(k_{\mu} U^{\mu}, T\right)$ is understood as a scalar, and $U^{\mu}$ is the 4-velocity of the thermo-system as measured in $F$. We have used $B\left(k_{\mu} U^{\mu}, T\right)$ instead of $P(\omega, T)$ to distinguish this version of the Planck distribution law from its conventional formulation[3, 4].

To see the transformation properties of $B\left(k_{\mu} U^{\mu}, T\right)$ in (1), consider a blackbody at rest and located at the origin of $F$. In another inertial frame $F^{\prime}$ moving with a relative velocity $V$ along the $+x$ axis relative to $F$, the 4-velocity $U^{\prime \mu}$ of the blackbody is,

$$
U^{\prime \mu}=\left(\frac{1}{\sqrt{1-V^{2}}}, \frac{-V}{\sqrt{1-V^{2}}}, 0,0\right),
$$

so that

$$
k_{\mu}^{\prime} U^{\prime \mu}=k_{0}^{\prime} U_{0}^{\prime}-k_{x}^{\prime} U_{x}^{\prime}=k_{0}^{\prime} \frac{\left[1+V \cos \theta^{\prime}\right]}{\sqrt{1-V^{2}}}=k_{\mu} U^{\mu}=k_{0},
$$

where $\cos \theta^{\prime}=k_{x}^{\prime} / k_{0}^{\prime}$. The relation (3) between $k_{0}$ and $k_{0}^{\prime}$ is the well-known relativistic Doppler shift. Writing the invariant Planck law (1) in terms of the non-invariant radiation energy $\omega^{\prime}=k_{0}^{\prime}$ and the 4-velocity (2) in $F^{\prime}$, we have

$$
B^{\prime}\left(k_{\mu}^{\prime} U^{\prime \mu}, T\right)=\frac{1}{\exp \left[\omega^{\prime}\left(1+V \cos \theta^{\prime}\right) /\left(k_{B} T \sqrt{1-V^{2}}\right)\right]-1} .
$$


In contrast, in the conventional formulation[3, 4], one accepts the original form of the Planck distribution, $P(\omega, T)=1 /\left[\exp \left(\omega / k_{B} T\right)-1\right]$, for a blackbody at rest in an inertial frame $F(t, x, y, z)$, which involves the quantity $\omega / k_{B} T=k_{0} / k_{B} T$. One reasons that since the zeroth component of the wave 4-vector $k^{\mu}=\left(\omega, k_{x}, k_{y}, k_{z}\right)$ transforms as $\omega=\omega^{\prime}\left[1+V \cos \theta^{\prime}\right] / \sqrt{1-V^{2}}, \cos \theta^{\prime}=k_{x}^{\prime} / k_{0}^{\prime}$ if the temperature transforms as

$$
T=T^{\prime}\left(\theta^{\prime}\right)\left(1+V \cos \theta^{\prime}\right) / \sqrt{1-V^{2}},
$$

then one has $\omega / k_{B} T=\omega^{\prime} / k_{B} T^{\prime}$. Therefore, the Planck's distribution $P(\omega, T)=1 /\left(\exp \left(\omega / k_{B} T\right)-1\right)$ has the same form in both $F$ and $F^{\prime}$ frames: $P(\omega, T)=P^{\prime}\left(\omega^{\prime}, T^{\prime}\right)$.

However, in this case, the Planck distribution law $P(\omega, T)$ is incompatible with the principle of relativity in that the laws of physics now do NOT have the same form in all inertial frames. This can be seen explicitly if one considers three frames $F, F^{\prime}$ and $F^{\prime \prime}$, the transformations of $P(\omega, T)$ and the ratio $\omega / k_{B} T$ in them. One can see that there is a preferred frame in which the Planck law $P(\omega, T)$ takes the simplest form, if the angle-dependent temperature (5) alone is used.

Furthermore, this conventional formulation with the angle dependent temperature (5) has two subtle conceptual difficulty. First, in special relativity, since $\omega$ is the zeroth component of a 4-vector, the ratio $\omega / k_{B} T=\omega^{\prime} / k_{B} T^{\prime}$ is invariant only if $k_{b} T$ is also the zeroth component of a 4-vector parallel to the $k^{\mu} 4$-vector, and what this 4-vector that involves the temperature might be is never fully specified in the conventional formulation. Instead, one simply introduces[3] equation (5) for the transformation of temperature rather than a temperature 4-vector, begging the question, just what kind of quantity is temperature? Since it is not invariant, it cannot be a scalar. Second, none of the Lorentz transformations of the temperature of a thermo-system, including those developed by Einstein[8], Planck[9], Ott[10], Arzelies[11], Landsberg[12], van Kampen[13] and others, have ever considered an angledependent transformation of temperature. As such, the angle-dependent temperature transformation in (5) is simply an ad hoc assumption and neither complete nor completely satisfactory.

Comparing the result (4) with the conventional formulation,

$$
P(\omega, T)=P^{\prime}\left(\omega^{\prime}, T^{\prime}\right)=1 /\left[\exp \left(\omega^{\prime}\left(1+V \cos \theta^{\prime}\right) /\left(k_{B} T \sqrt{1-V^{2}}\right)\right)-1\right],
$$

with the angle-dependent temperature (5), one can see that the two are quantitatively identical. However, they have an important conceptual and physical difference.

First, there was no need to construct a new temperature four-vector with an unusual angledependent temperature transformation.

Second, because $k_{\mu} U^{\mu}$ and $k_{B} T$ are now separately Lorentz invariant and $k_{B} T$ is a Lorentz scalar, if the invariant blackbody distribution $B\left(k_{\mu} U^{\mu}, T\right)$ and the CMB temperature are isotropic in one frame $F$, then they will both be isotropic in every other inertial frame as well.

\section{Generalized Planck distribution for non-inertial frames}

Actually, we can go further towards the non-inertial frame. In the literature, there exists a fundamental space-time symmetry framework called limiting four-dimensional symmetry (or limiting LorentzPoincaré invariance) that can help us generalize physical laws in inertial frames to non-inertial frames of reference.[1, 2] In particular, one can use this framework to derive a set of coordinate transformations between an inertial frame $F^{\prime}$ and a rotating frame $F_{r}$ that (a) are exact, (b) simplify to the Lorentz transformations in the appropriate limit (i.e., as the orbital radius goes to infinity while the product of the orbital radius and angular velocity remains finite[15]), (c) are consistent with the results of experiments such as the Davies-Jennison experiment, Thim's experiment involving radio sources in circular motion[2,15], and high energy experiments involving unstable particles in a circular storage ring, and (d) support the analysis of the Wilson experiment.[7] These transformations can also 
give us the metric tensors $P_{\mu \nu}(x)$ necessary for generalizing the blackbody distribution to rotating and constant-linear-acceleration frames.

To investigate the effect of the Earth's orbital motion on the blackbody distribution (1), for simplicity, let us ignore the relative linear motion between the solar system and the (possible) 'CMB rest frame' and approximate the Earth's orbital motion as a circular orbit. Consider an inertial frame $F^{\prime}\left(t^{\prime}, x^{\prime}, y^{\prime}, z^{\prime}\right)$ and a rotational frame $F_{r}\left(t_{r}, x_{r}, y_{r}, z_{r}\right)$, where the origin of $F_{r}$ orbits the origin of $F^{\prime}$ at a constant distance $R$ with a constant angular velocity $\Omega \equiv \Omega_{r}$ (as measured in the rotating frame). A Cartesian coordinate system is used in both frames, set up in such a way that the positive portion of the $y_{r}$-axis of the frame $F_{r}$ always extends through the origin of $F^{\prime}$. Let the Earth be at rest relative to the rotating frame and located at $x_{r}=\rho, y_{r}=0, z_{r}=0$. The exact rotational coordinate transformations are then [15]

$$
\begin{gathered}
t^{\prime}=\gamma\left(t_{r}+\boldsymbol{\rho} \cdot \boldsymbol{\beta}\right), \quad x^{\prime}=\gamma\left[x_{r} \cos \left(\Omega t_{r}\right)-\left(y_{r}-R\right) \sin \left(\Omega t_{r}\right)\right], \\
y^{\prime}=\gamma\left[x_{r} \sin \left(\Omega t_{r}\right)+\left(y_{r}-R\right) \cos \left(\Omega t_{r}\right)\right], \quad z^{\prime}=z_{r}, \\
\beta=|\boldsymbol{\Omega} \times \mathbf{S}|=\Omega \sqrt{x_{r}^{2}+\left(y_{r}-R\right)^{2}}=\Omega S<1, \quad \boldsymbol{\rho} \cdot \boldsymbol{\beta}=x_{r} R \Omega, \quad \gamma=\frac{1}{\sqrt{1-\beta^{2}}}, \\
\boldsymbol{\rho}=\left(x_{r}, y_{r}, 0\right), \quad \mathbf{S}=\left(x_{r}, y_{r}-R, 0\right), \quad \boldsymbol{\beta}=\mathbf{\Omega} \times \mathbf{S}, \quad \mathbf{\Omega}=(0,0, \Omega) .
\end{gathered}
$$

The times $t^{\prime}$ and $t_{r}$ are measured in units of length. ${ }^{2}$ The rotational transformation (6) form a pseudogroup (in the sense of Whitehead and Veblen)[17]. In the limit $R \rightarrow \infty$ and $\Omega \rightarrow 0$ such that the product $R \Omega=\beta_{o}$ is a finite non-zero constant velocity, the transformations (6) with (7) reduce to the exact Lorentz transformations.

For simplicity of discussions below, it suffices to set $R=0$ in (6). To the first order in $\rho \Omega$, the non-vanishing components of the covariant metric tensors $P_{r \mu v}(x)$ of the rotating frame $F_{r}$ are then [14],

$$
P_{r 00}=-P_{r 11}=-P_{r 22}=-P_{r 33}=1, \quad P_{r 01}=\Omega y_{r}, \quad P_{r 02}=-\Omega x_{r} .
$$

In this approximation, the contravariant metric tensors $P_{r}^{\mu v}$ are the same, e.g., $P_{r}^{01}=\Omega y_{r}, P_{r}^{02}=-\Omega x_{r}$, etc.

The covariant wave vectors for the blackbody radiation in the inertial and rotating frames are denoted by $k_{\mu}^{\prime}=\left(k_{o}^{\prime}, k_{1}^{\prime}, k_{2}^{\prime}, k_{3}^{\prime}\right)$ and $k_{r \mu}=\left(k_{r 0}, k_{r 1}, k_{r 2}, k_{r 3}\right)$, respectively. They are related by the rotational wave-vector transformations, [15]

$$
\begin{gathered}
k_{0}^{\prime} \approx k_{r 0}-\Omega \rho k_{r 2}, \quad k_{1}^{\prime} \approx k_{r 1} \cos \left(\Omega t_{r}\right)-k_{r 2} \sin \left(\Omega t_{r}\right), \\
k_{2}^{\prime} \approx k_{r 1} \sin \left(\Omega t_{r}\right)+k_{r 2} \cos \left(\Omega t_{r}\right), \quad k_{3}^{\prime}=k_{r 3} .
\end{gathered}
$$

For purposes of making predictions in $F^{\prime}$ and $F_{r}$ frames, we shall concentrate on a simple case, where $k_{2}^{\prime}=k_{3}^{\prime}=0$. The quantity $k_{r \mu}$ denotes the corresponding wave vector as measured in $F_{r}$ at the location of the detector, $\left(x_{r}, y_{r}, x_{r}\right)=(\rho, 0,0)$. In this case, (9) and $k_{0}^{\prime}=k_{1}^{\prime}>0$ lead to the relations

$$
\frac{k_{r 2}}{k_{r 0}} \approx \frac{-\sin \left(\Omega t_{r}\right)}{1-\Omega \rho \sin \left(\Omega t_{r}\right)}, \quad k_{r 0} \approx \frac{k_{0}^{\prime}}{1+\Omega \rho \sin \left(\Omega t_{r}\right)} .
$$

While $k_{0}^{\prime}$ and $k_{1}^{\prime}$ are time-independent, $k_{r 0}, k_{r 1}$ and $k_{r 2}$ measured in $F_{r}$ are time-dependent.

\footnotetext{
${ }^{2}$ The operational meaning of space-time coordinates in $F^{\prime}\left(t^{\prime}, x^{\prime}, y^{\prime}, z^{\prime}\right)$ is well-known. The operational meaning of coordinates in a non-inertial frame such as $F_{r}\left(t_{r}, x_{r}, y_{r}, z_{r}\right)$ can be realized by a grid of (computerized) 'space-time clocks' [16] located in $F_{r}$ and satisfies (6).
} 
Now consider a thermo-system at rest in $F^{\prime}$ with $U^{\prime \mu}=(1,0,0,0)$. To first order in $\rho \Omega$, its velocity in $F_{r}$ is $U_{r}^{\mu}=d x_{r}^{\mu} / d s \approx\left(1, \Omega y_{r},-\Omega x_{r}, 0\right)$. Thus, we have the relationship,

$$
P_{r}^{\mu v} k_{r \mu} U_{r v} \approx k_{r 0}\left[1+\rho \Omega \sin \left(\Omega t_{r}\right)\right],
$$

for the rotational frame $F_{r}$, where $\rho \Omega$ is the Earth's orbital velocity, and we have used $U_{r}^{\mu}=P_{r}^{\mu v} U_{r v}$ and (10).

In general, one can use the metric tensor $P^{\mu v}$ in place of the Minkowski metric tensor $\eta^{\mu v}$ in order to generalize the invariant blackbody distribution (1) from an inertial frame to a non-inertial frame, such as a constant-linear-acceleration (CLA) frame $F_{C L A}$ or a constant rotational frame $F_{r}$.

(A) Constant-linear-acceleration frame $F_{C L A}$ :

$$
B_{C L A}\left(k_{\lambda} U^{\lambda}, T\right)=\frac{1}{\left[\exp \left(P^{\mu v} k_{\mu} U_{v}\right) /\left(k_{B} T\right)-1\right]},
$$

where $P_{00}=\gamma^{2}\left(\gamma_{o}^{2}-\alpha_{o} x\right), P_{11}=P_{11}=P_{22}=-1 ;[16]$ and

(B) Rotational frame $F_{r}$ with constant angular velocity:

$$
B_{r}\left(k_{r \lambda} U_{r}^{\lambda}, T\right)=\frac{1}{\left[\exp \left(P_{r \mu \nu} k_{r}^{\mu} U_{r}^{v}\right) /\left(k_{B} T\right)-1\right]},
$$

where $P_{r \mu \nu}$ is given by (8).

To the first order in $\rho \Omega$, we have the generalized blackbody distribution for a rotational frame,

$$
B_{r}\left(k_{r \lambda} U_{r}^{\lambda}, T\right) \approx \frac{1}{\left[\exp \left(k_{0}^{\prime} / k_{B} T\right)-1\right]}, \quad T=T_{i n v},
$$

where $k_{r \lambda} U_{r}^{\lambda}=P_{r}^{\lambda v} k_{r \lambda} U_{r v} \approx k_{r 0}\left[1+\rho \Omega \sin \left(\Omega t_{r}\right)\right] / k_{B} T$, and we have used equations (10) and (11). As expected, the GF covariant blackbody distribution law (14) does not depend on time.

Therefore, based on (1), (10) and (14), when the GF covariant distribution law (14) is measured in a rotational frame $F_{r}$, we obtain the time-independent blackbody distribution law,

$$
B_{r}\left(k_{r \lambda} U_{r}^{\lambda}, T\right)=B^{\prime}\left(\eta^{\mu v} k_{\mu}^{\prime} U_{v}^{\prime}, T\right),
$$

where we have used the relation $P_{r}^{\alpha \beta} k_{r \alpha} U_{r \beta}=\eta^{\alpha \beta} k_{\alpha}^{\prime} U_{\beta}^{\prime}$.

\section{Non-invariant spectral radiance and six-month variation}

However, we stress that satellite experiments do not measure the GF covariant distribution law $B_{r}\left(P_{r}^{\mu \nu} k_{r \mu} U_{r v}, T\right)$. Instead, they measure the non-invariant spectral radiance in frequency (or power per unit area) $W_{r}$, which is the power emitted per unit projected area of a blackbody at temperature $T$, into a unit solid angle, in the interval $k_{r}$ to $k_{r}+d k_{r}, k_{r}=\left|\mathbf{k}_{r}\right|>0$. In the $F_{r}\left(t_{r}, x_{r}, y_{r}, z_{r}\right)$ frame with curvilinear coordinates, the spectral radiance $W_{r}$ is related to the covariant differential of the photon energy-momentum tensor $D T_{r \mu \nu}$,

$$
\begin{gathered}
D T_{r \mu \nu}=\frac{2}{\pi^{2}} k_{r \mu} k_{r v} B_{r}\left(k_{r \lambda} U_{r}^{\lambda}, T\right) \delta\left(P_{r}^{\alpha \beta} k_{r \alpha} k_{r \beta}\right) \sqrt{-\operatorname{det} P_{r}^{\lambda \sigma}} d^{4} k_{r}, \\
W_{r}=D T_{r 00} /\left(d k_{r} d \bar{\Omega}_{r}\right), \quad k_{r}=\left|\mathbf{k}_{r}\right|
\end{gathered}
$$


where the integration over $k_{r 0}>0$ in (16) is understood and $\bar{\Omega}_{r}$ is the solid angle. To carry out this integration in the general case, $\delta\left(f\left(k_{r 0}, \mathbf{k}_{r}\right)\right) d k_{r 0}$, one may use

$$
f\left(k_{r 0}, \mathbf{k}_{r}\right)=P_{r}^{\alpha \beta} k_{r \alpha} k_{r \beta}=\eta^{\alpha \beta} k_{\alpha}^{\prime} k_{\beta}^{\prime}
$$

and (9) to obtain

$$
f\left(k_{r 0}, \mathbf{k}_{r}\right)=\left(k_{r 0}-\Omega \rho k_{r 2}\right)^{2}-k_{r}^{2}
$$

to the first order in $\rho \Omega$. Thus, we have

$$
D T_{r 00}=\left[\frac{2}{\pi^{2}} k_{0} k_{0} B_{r}\left(k_{r \lambda} U_{r}^{\lambda}, T\right) \frac{d^{3} k_{r}}{2 k_{r}}\right]_{k_{r 0}=k_{r}+\rho \Omega k_{r 2}}
$$

To see explicitly the six-month variation in the measured $W_{r}$, it suffices to consider the simple case $k_{\mu}^{\prime}=\left(k_{0}^{\prime}, k_{1}^{\prime}, 0,0\right)$ at the location of the detector $\left(x_{r}, y_{r}, z_{r}\right)=(\rho, 0,0)$ using relation (12). Based on (16), (17) and the corresponding relation $d T_{00}^{\prime}=W^{\prime} d k^{\prime} d \bar{\Omega}^{\prime}$ for the inertial frame $F^{\prime}$, we obtain the following relationship for spectral radiances measured in the two frames,

$$
\frac{W_{r}}{W^{\prime}} \approx\left(\frac{\omega_{r}}{\omega^{\prime}}\right)^{3}\left[1+\Omega \rho \sin \left(\Omega t_{r}\right)\right] \approx 1-2 \Omega \rho \sin \left(\Omega t_{r}\right),
$$

where we have used the rotational frequency shift in (9) and the invariance of the blackbody distributions (14) and (4). For Earth's orbital motion, we may consider the Earth to be at the location of the detector, $\left(x_{r}, y_{r}, x_{r}\right)=(\rho, 0,0)$ in the rotational frame $F_{r}$. Thus we have

$$
\Omega \approx 0.2 \times 10^{-6} / \mathrm{sec} \quad \text { and } \quad \Omega \rho \approx 9.9 \times 10^{-5}
$$

(in natural units) for (19).

Therefore, we predict that the blackbody spectral radiance $W_{r}$ measured from the orbiting Earth will indeed display a six-month variation (19), consistent with the qualitative results of satellite experiments. What remains now is to test the quantitative predictions of (19) against satellite experiments measuring the CMB.

\section{Big Jets model and the missing half of the antimatter universe}

According to the Big Jets model[16, 18], the presence of an extremely distant 'antimatter-galaxies blackbody' could contribute to the observed dipole anisotropy in CMB. The Big Jets model suggests that the universe began with two Big Jets rather than one Big Bang, similar to the particle collision phenomena one might encounter in high-energy laboratories. The model suggests that the universe originated with the formation of two diametrically opposed jets, composed of baryons and anti-baryons, etc. in each jet. We may picture these two jets as two big fire balls moving away from each other. The processes of their annihilations and decays eventually lead to a baryon-electron dominated fireball and an antibaryon-positron dominated fireball. Then, from the vantage point of an observer in either fireball, the evolution of that 'observer's fireball' would be similar to the general features of a hot Big Bang. Now the two gigantic fire balls cool down to become one baryon-electron dominated blackbody (called 'Yang blackbody') and a antibaryon-positron dominated blackbody (called 'Yin blackbody') with a temperature of roughly $3 \mathrm{~K}$, which are separated by an extremely large distance $D_{Y Y}$. As a result, lights emitted from anti-supernovae and anti-galaxies are too faint to be detected. Only the microwaves emitted from the Yin blackbody as a whole may be detected by our apparatus. 
To search the missing half of the universe suggested by the Big Jets model, let us approximate, for simplicity, blackbodies Yin and Yang as two gigantic spheres of radius $R_{Y}$. Microwaves emitted from the antimatter Yin blackbody toward our Yang blackbody are completely absorbed, causing the Yang hemisphere facing Yin to be slightly warmer than the opposite hemisphere and giving the Yang blackbody temperature a 'pseudo-dipole distribution' roughly. Suppose the Yang blackbody receiving microwaves from the Yin blackbody so that its hemisphere facing Yin is warmer by $\Delta T$, depending on angle. One may try to estimate $\Delta T$ by using the Stefan-Boltzmann law and the condition of equilibrium. But it will involve some unknown parameters. Thus, we just use $\Delta T$ as a parameter to be determined by experiments. Suppose $D_{Y Y}>>D_{Y}$, the Big Jets model implies that, approximately, the observed temperature $T_{o b}$ in our Yang blackbody has a simple angle dependence,

$$
T_{o b} \approx T_{C M B}+Y(\theta) \Delta T
$$

$$
\begin{aligned}
& Y(\theta)=\cos \theta, \text { for the surface of the hemisphere toward Yin blackbody, } \\
& Y(\theta)=0, \text { for the surface of the hemisphere away from Yin blackbody, }
\end{aligned}
$$

where $\theta=0$ corresponds to the direction from the center of Yang blackbody to that of Yin blackbody.

The reason for $Y(\theta)=0$ in (20) is that the (parallel) microwaves from the Yin blackbody are totally absorbed by the Yang blackbody, so that the temperature on the Yang hemisphere away from the Yin blackbody remains uniform, $T_{o b}=T_{C M B}$, independent of angles.

In the presence of the Yin blackbody, the spectral radiance $W_{B}^{\prime}$ of the blackbody radiation measured in the inertial frame $F^{\prime}$ involves the Lorentz invariant temperature $T$ and the invariant blackbody distribution $B^{\prime}\left(k_{\lambda}^{\prime} U^{\prime \lambda}, T_{o b}\right)$. The non-invariant spectral radiance $W_{B}^{\prime}\left(k_{\lambda}^{\prime} U^{\prime \lambda}, T_{o b}\right)$ can be approximated by the Taylor series expansion in the inertial frame $F^{\prime}$. We have

$$
\begin{gathered}
W_{B}^{\prime}\left(k_{\lambda}^{\prime} U^{\prime \lambda}, T_{o b}\right)=\frac{1}{4 \pi^{3}}\left(k_{0}^{\prime}\right)^{3} B^{\prime}\left(k_{\lambda}^{\prime} U^{\prime \lambda}, T_{o b}\right) \\
\approx W_{B}^{\prime}\left(k_{\lambda}^{\prime} U^{\prime \lambda}, T_{C M B}\right)+W_{B J}^{\prime},
\end{gathered}
$$

where the second term $W_{B J}^{\prime}$ in (21) determines the CMB dipole spectrum due to the blackbody Yin in the Big Jets model. We obtain the pseudo-dipole spectrum due to the antimatter blackbody,

$$
\begin{gathered}
W_{B J}^{\prime} \approx \Delta T \cos \theta\left(\frac{\partial W_{B}^{\prime}\left(k_{\lambda}^{\prime} U^{\prime \lambda}, T_{o b}\right)}{\partial T_{o b}}\right)_{T_{o b}=T_{C M B}} \\
\approx \Delta T \cos \theta\left(\frac{\omega^{\prime 4}}{4 \pi^{3} k_{B} T_{C M B}^{2}} \frac{\left(\exp \left(\omega^{\prime} / k_{B} T_{C M B}\right)\right.}{\left[\exp \left(\omega^{\prime} / k_{B} T_{C M B}\right)-1\right]^{2}}\right),
\end{gathered}
$$

where we have taken the non-relativistic limit of the 4-velocity $U^{\prime \lambda}$, i.e., $U^{\prime \lambda}=(1,0,0,0)$, and $k_{\sigma}^{\prime} U^{\prime \sigma}=k_{0}^{\prime}=\omega^{\prime}$.

For the sake of discussion, suppose that anti-matter blackbody happened to be in the direction of the constellation Leo, then the prediction (22) by the Big-Jets model turns out to be the same shape as the frequency spectrum of the conventional theory[3, 19] and, hence, both are consistent with the experiment of Fixsen et al.[19] On the other hand, when one measures the spectral radiance $\left.W_{B}^{\prime}\left(k_{\lambda}^{\prime} U^{\prime \lambda}, T\right)\right)$ in (21) in the direction away from Leo, the second term $W_{B J}^{\prime}$ in (21) does not contribute because $Y\left(\theta^{*}\right)=0$ in (21) due to the complete absorption of the microwaves by our blackbody Yang. Thus, in the direction away from the antimatter blackbody Yin, (20) and (21) lead to the usual CMB spectral radiance,

$$
W_{B}^{\prime}\left(k_{\lambda}^{\prime} U^{\prime \lambda}, T\right)=W_{B}^{\prime}\left(k_{\lambda}^{\prime} U^{\prime \lambda}, T_{C M B}\right)
$$


which is independent of the angle $\theta$. The result (23) hold for the surface of the hemisphere of the Yang blackbody away from the Yin blackbody and suggests a new method to search for the missing half of the antimatter universe, as we shall discuss below.

In contrast to (22) and (23), the conventional theory with angle-dependent temperature (5) leads to the spectrum

$$
\begin{gathered}
B_{v}^{\prime}\left(T^{\prime}\right) \approx B_{v}^{\prime}\left(T_{C M B}\right)+V T_{C M B} \cos \theta^{\prime}\left(\frac{\partial B_{v}^{\prime}\left(T^{\prime}\right)}{\partial T^{\prime}}\right)_{T^{\prime}=T_{C M B}}, \\
T^{\prime} \approx T_{C M B}\left[1+V \cos \theta^{\prime}\right],
\end{gathered}
$$

where $\theta^{\prime}$ is the angle between the direction of the motion of the solar system and the line of sight. The velocity $V \approx 370 \mathrm{~km} / \mathrm{s}$ (in MKS units) is interpreted as the motion of the solar system ( $F^{\prime}$ frame) in the CMB. The spectrum (or the Planck function) $B_{v}(T)$ takes the simplest form $\omega^{3} /\left[\exp \left(\omega /\left(k_{B} T\right)\right)-1\right]$, $\omega=2 \pi v$ in the preferred frame $F[20,21]$.

\section{Discussions}

The conclusion based on making the blackbody distribution laws (1) and (4) explicitly invariant does not imply that the conventional theory with (24) and the angle-dependent temperature (5) is wrong because they use different definitions (or 'interpretations') of the temperature. Nevertheless, from the viewpoint of Lorentz-Poincare invariance, the dipole anisotropy in CMB temperature in the conventional theory with (24) is due to an unnatural interpretation of the invariant distribution law $B^{\prime}\left(k_{\lambda}^{\prime} U^{\prime \lambda}, T\right)$ given by (4), in terms of the non-invariant variable $\omega^{\prime}$ and an ad hoc angle-dependent temperature (5), i.e. $1 / T^{\prime}\left(\theta^{\prime}\right)=\left(1+V \cos \theta^{\prime}\right) /\left(T \sqrt{1-V^{2}}\right)$. This can be seen from the fact that the Lorentz invariant law $B^{\prime}\left(k_{\lambda}^{\prime} U^{\prime \lambda}, T\right)$ in (4) turns out to be quantitatively identical to $P^{\prime}\left(\omega^{\prime}, T^{\prime}\right)$,

$$
B^{\prime}\left(k_{\lambda}^{\prime} U^{\prime \lambda}, T\right)=P^{\prime}\left(\omega^{\prime}, T^{\prime}\right)=1 /\left[\exp \left(\omega^{\prime}\left(1+V \cos \theta^{\prime}\right) /\left(k_{B} T \sqrt{1-V^{2}}\right)\right)-1\right]
$$

Thus, the same set of satellite data can be viewed from two conceptually different viewpoints. We believe that the Lorentz invariant view of the distribution law $B^{\prime}\left(k_{\lambda}^{\prime} U^{\prime \lambda}, T\right)$ in terms of the scalar function $k_{\lambda}^{\prime} U^{\prime \lambda}=k_{\lambda} U^{\lambda}$ and the invariant temperature $T=T_{i n v}$ is more natural. The reasons are that the blackbody distribution law $B^{\prime}\left(k_{\lambda}^{\prime} U^{\prime \lambda}, T\right)$ satisfies the principle of relativity and that the invariant temperature (or $K_{B} T$ ) is consistent with those developed by Einstein et al for relativistic thermodynamics. [8-13]. On the contrary, if one considers (24) and the angle-dependent temperature (5) in three inertial frames explicitly, one sees that they are not consistent with the principle of relativity in that the ad hoc temperature (5) itself does not have proper transformation properties within the 4-dimensional symmetry framework.

Based on the general-frame covariant blackbody distributions (1), (4), (12)-(15), it is unnatural and inconsistent with the principle of relativity to attribute the 'dipole anisotropy of the CMB' to the motion of the solar system relative to the CMB. The general-frame covariant result (15) implies that if the Lorentz invariant distribution law $B\left(k_{\mu}^{\prime} U^{\prime \mu}, T\right)$ and the CMB temperature are isotropic in one inertial frame $F^{\prime}$, then they will both be isotropic in every inertial and rotational frames as well, according to the principle of limiting Lorentz-Poincaré invariance.

For experimental tests and search for the missing half of the universe, the Big Jets model predicts that the physical universe is made of two gigantic blackbodies with the following properties:

(i) Within our 'matter blackbody', there are no 'antimatter stars' or 'anti-matter galaxies' due to annihilation processes in the early epoch of evolution. This has been supported by observations and experiments in our 'matter blackbody.' 
(ii) It is remarkable that the dipole spectrum (22) of the antimatter blackbody has the same shape as that of the usual 'hot dipole' in the direction of the constellation Leo. Thus, it is interesting to see whether evidence for the missing half of the universe may be hidden in the data of the hot dipole anisotropy. Their spectra differ only in the 'cold dipole', as shown in (23). Therefore, the Big Jets model suggests that, in general, one should examine whether there is a small asymmetry of the spectrum in two opposite directions in the sky, towards and away from the constellation Leo. Such a small asymmetry could be tested by, say, the experiment of Fixsen et al[19], which measured the specific spectrum of the dipole for approximately $60 \%$ of the sky (including the 'hot pole', $\cos \theta>0){ }^{3}$

The work was supported in part by the Jing Shin Research Fund of the UMass Dartmouth Foundation.

\section{References}

[1] J. P. Hsu and L. Hsu, Phys. Letters, A 196, 1 (1994). Such a broad formulation of space-time transformations based solely on the first postulate is essential for generalization to non-inertial frames, especially the rotational frame for our discussion here.

[2] J. P. Hsu and L. Hsu, Eur. Phys. J. Plus, 128, 74 (2013), arXiv: 1307.0662.

[3] D. Scott and G. F. Smoot, "27. COSMIC MICROWAVE BACKGROUND”, section 27.2.2, for The Review of Particle Physics, K.A. Olive et al. (Particle Data Group), Chinese Phys. C 38, 09000, (2014); (http://pdg.lbl.gov). See also ref. 21.

[4] S. Weinberg, Gravitation and Cosmology, Principles and Applications of the General Theory of Relativity (John Wiley and Sons, 1972) pp. 520-522.

[5] J. P. Hsu and L. Hsu, in the Proceedings of the Twelfth International Conference on Gravitation, Astrophysics and Cosmology, Moscow, 2015 (Ed. V. Melnikov and J. P. Hsu, World Scientific, 2016) p. 3; and reference therein.

[6] L. Hsu and J. P. Hsu, 'Experiments on Invariance of Planck's Law and Anisotropy of Cosmic Microwave Background' (UMassD preprint, 2017).

[7] G. N. Pellegrini and A. R. Swift, Am. J. Phys. 63, 694 (1995) and references therein.

[8] A. Einstein, Jahrb. Rad. u. Elektr, 4,411 (1907).

[9] M. Planck, Ann. d. Phys. 26, 792 (1908).

[10] H. Ott, Zeitschr. d. Phys. 174, 70 (1963).

[11] H. Arzelies, Nuovo Cimento 35, 792 (1965).

[12] P.T. Landsberg, Nature 212, 571 (1966).

[13] N.G. van Kampen, Phys. Rev. 173, 295 (1968).

[14] L. Landau and E. Lifshitz, The Classical Theory of Fields, (Addison-Wesley, 1951), p. 31.

[15] L. Hsu and J. P. Hsu, Eur. Phys. J. Plus, 129, 16 (2014).

[16] J. P. Hsu and L. Hsu, Space-Time Symmetry and Quantum Yang-Mills Gravity (Singapore, World Scientific) pp. 26-28; p. 225, note 20.

[17] O. Veblen and J. H. C. Whitehead, The Foundation of Differential Geometry (Cambridge Press, 1953), pp. 37-38.

\footnotetext{
${ }^{3}$ At present, the dipole spectrum has been fit almost to the entire sky (with the exception of about $1 \%$ of the sky missing due to the lack of FIRAS data there and another $(10-20) \%$ of the sky being ignored because the galactic plane is too bright and difficult to normalize.[22]). Fixsen has performed a quick fit separately to the hot pole (where $\cos \theta>0$ ) and the cold pole (where $\cos \theta<0$ ), then looked for differences between the two spectra. There is no difference to a sensitivity of a few $\times 10^{-4}$ relative to the peak of the CMB. One may use a simple property of a maximum related to the energy density of radiation to roughly estimate the corresponding temperature difference between two opposite directions in the sky as $\Delta T<3 \times 10^{-4} \mathrm{~K}$, due to the possible presence of a distant antimatter blackbody.
} 
[18] J. P. Hsu, Chin. J. Phys. bf 41, 015101 (2017).

[19] D. J. Fixsen, et al, Astrophys. J. 420, 445 (1994).

[20] G. F. Smoot, Nobel Lecture, (https://www.nobelprize.org/nobel_prizes/physics/laureates/2006/smootlecture.html) pp.123-124.

[21] V. S. Weisskoph, Am. Sci. 71473 (1983).

[22] D. J. Fixsen, private correspondence, 2017. 